\title{
Diacronie
}

Studi di Storia Contemporanea

$N^{\circ} 32,4 \mid 2017$

Proiezioni individuali e agire collettivo nella storia

\section{Dalla public history alla applied history}

Ruolo pubblico e funzione politica della storia nel recente dibattito storiografico angloamericano

\section{Benedetta Giuliani}

\section{OpenEdition}

\section{Edizione digitale}

URL: http://journals.openedition.org/diacronie/6473

DOI: 10.4000/diacronie.6473

ISSN: 2038-0925

Editore

Association culturelle Diacronie

\section{Notizia bibliografica digitale}

Benedetta Giuliani, « Dalla public history alla applied history », Diacronie [Online], № 32, 4 | 2017,

documento 1, Messo online il 29 décembre 2017, consultato il 01 mai 2019. URL : http://

journals.openedition.org/diacronie/6473; DOI : 10.4000/diacronie.6473 


\title{
Diacronie
}

Studi di Storia Contemporanea

\section{$32,4 / 2017$}

Proiezioni individuali e agire collettivo nella storia. Ruoli sociali, aspetti politici e nodi storiografici tra pubblico e privato

\section{Dalla public history alla applied history. Ruolo pubblico e funzione politica della storia nel recente dibattito storiografico angloamericano}

\author{
Benedetta GIULIANI
}

Per citare questo articolo:

GIULIANI, Benedetta, «Dalla public history alla applied history. Ruolo pubblico e funzione politica della storia nel recente dibattito storiografico angloamericano», Diacronie. Studi di Storia Contemporanea : Proiezioni individuali e agire collettivo nella storia. Ruoli sociali, a spetti politici e nodi storiografici tra pubblico e privato, 32, 4/2017, 29/12/2017,

URL: < http://www.studistorici.com/2017/12/29/giuliani_numero_32/ >

Diacronie Studi di Storia Contemporanea $\rightarrow$ http://www.diacronie.it

Rivista storica online. Uscita trimestrale.

redazione.diacronie@hotmail.it

Comitato di direzione: Naor Ben-Yehoyada - João Fábio Bertonha - Christopher Denis-Delacour - Maximiliano Fuentes Codera Anders Granås Kjøstvedt - John Paul Newman - Deborah Paci - Niccolò Pianciola - Spyridon Ploumidis - Wilko Graf Von Hardenberg

Comitato di redazione: Jacopo Bassi - Luca Bufarale - Gianluca Canè - Fausto Pietrancosta - Alessandro Salvador - Matteo Tomasoni - Luca Zuccolo 


\title{
1/ Dalla public history alla applied history. Ruolo pubblico e funzione politica della storia nel recente dibattito storiografico angloamericano
}

\author{
Benedetta GIULIANI
}

\begin{abstract}
Negli ultimi tempi i rapporti tra le discipline umanistiche e le moderne società post-industriali non sono felici. Declassati a rango di ludus intellettuali molti di questi saperi, storia inclusa, cercano di ribadire la propria legittimità scientifica e di collocarsi nella società e nel mercato. Per ciò che concerne la storiografia, nel tentativo di reagire a questa crisi di ruolo e di significato, alcuni storici hanno sviluppato percorsi di ricerca capaci di dare risposte ambiziose circa la funzione dell'analisi storica nel mondo odierno, sottolineando soprattutto come la storia possa contribuire a migliorare la prassi di governo. L'articolo intende esaminare i percorsi di ricerca più innovativi teorizzati dalla storiografia anglo-americana, ovvero la public history e, in particolare, la applied history. In relazione a quest'ultima verranno esaminate le attività del network di ricerca History \& Policy e dell'Applied History Project del Belfer Center.
\end{abstract}

\section{Introduzione}

Il lettore appassionato di politica internazionale avrà notato che, dalla fine degli anni Ottanta in poi, capi di Stato e ministri dei Paesi occidentali hanno spesso auspicato la creazione di un piano Marshall per risolvere le crisi economiche e umanitarie in Africa ${ }^{1}$. Concepiti di volta in volta come imponenti stanziamenti di risorse finanziarie o come programmi di prestiti agevolati, tali iniziative condividevano soprattutto il fatto di presentarsi come epigoni del grande piano di aiuti

\footnotetext{
${ }^{1}$ Cfr. PLAUT, Martin, «Revealed: Margaret Thatcher's "Marshall Plan for Southern Africa"», in The Conversation, 30 dicembre 2016, URL: < https://theconversation.com/revealed-margaret-thatchersmarshall-plan-for-southern-africa-70541 > [consultato il 1 novembre 2017]; CHÂTELOT, Christophe, «Il faut un plan Marshall pour électrifier l'Afrique», in Le Monde, 3 marzo 2015, URL: < http://www.lemonde.fr/afrique/article/2015/03/03/il-faut-un-plan-marshall-pour-electrifier-lafrique_4586493_3212.html > [consultato il 22 marzo 2017]; HUBBARD, Glenn, «Think Again: A Marshall Plan for Africa», in Foreign Policy, 13 agosto 2009, URL: < http://foreignpolicy.com/2009/08/13/think-again-amarshall-plan-for-africa/> [consultato il 1 novembre 2017].
} 
preparato dagli Stati Uniti per sostenere la rinascita economica dell'Europa nel secondo dopoguerra. Perennemente annunciati e mai pervenuti in una forma organica e coerente, i vari piani Marshall per l'Africa non hanno avuto lo stesso impatto dell'operazione a cui si ispiravano. Se lo European Recovery Program ha avuto il merito, tra le altre cose, di innovare profondamente le pratiche e l'idea stessa di foreign aid, le repliche pensate per il continente africano si sono rivelate finora aiuti ingenti ma disorganici, nobilitati dal riferimento a un termine storico di immediato valore simbolico quale appunto il piano Marshall. Elencare le ragioni che hanno determinato il loro insuccesso non rientra nelle mie intenzioni, mentre risulta possibile, nonché utile ai fini dell'articolo, sottolineare una fondamentale differenza di impostazione tra il piano Marshall originale e i suoi successori e considerare perché questo nome sia stato impiegato per descrivere piani di assistenza molto diversi dal progetto di ricostruzione europea.

Secondo Ernest May e Richard Neustadt una delle ragioni alla base del successo dello European Recovery Program (ERP) fu la particolare sensibilità storica di George Marshall, come pure di George Kennan e Dean Acheson, i quali elaborarono le proprie analisi sui futuri equilibri europei alla luce dei rapporti di forza e delle rivalità esistenti nel continente europeo negli anni dell'anteguerra ${ }^{2}$. Nell'articolare il piano di ricostruzione europea Marshall e i funzionari del dipartimento di Stato tennero bene a mente alcune considerazioni che la recente storia europea consigliava di non sottovalutare. La rivalità franco-tedesca e il timore di un ritorno dell'Europa al protezionismo del periodo pre-bellico, il quale non solo avrebbe danneggiato gli interessi dell'economia americana ma avrebbe anche reso più complicato estinguere la competitività tra grandi potenze, erano solo alcune delle principali lezioni che i pianificatori americani trassero dall'analisi del passato europeo. Secondo Neustadt e May, dunque:

[Marshall's] choices almost surely emerged from his looking toward the future with a long look at the past [...] What Marshall proposed was unprecedented in both character and scale. It was breathtakingly imaginative. Yet underneath was cautious concern about what might go wrong, and underneath that [...] was a deep awareness of long-running currents in both American and European experiences ${ }^{3}$.

La capacità di guardare al passato per individuare continuità e mutamenti all'interno del processo storico, ovvero quella che May e Neustadt definivano prospettiva storica, è ciò che distingue l'ERP dai programmi di aiuto ad esso precedenti e successivi. Eppure gli statisti contemporanei hanno continuato ad utilizzare il termine piano Marshall in riferimento a

\footnotetext{
${ }^{2}$ NEUSTADT, Richard, MAY, Ernest, Thinking in Time. The Uses of History for Decision Makers, New York, Free Press, 1986, p. 605.

${ }^{3}$ Ibidem, pp. 610-611.
} 
politiche di assistenza indirizzate a realtà economico-politiche prive di quelle condizioni che avevano permesso all'Europa post-bellica di impiegare costruttivamente gli aiuti d'oltreoceano.

L'uso attualizzato del piano Marshall non è che un esempio di quanto l'osservatore contemporaneo, sia esso un cittadino ordinario o un funzionario di governo, impieghi dei referenti storici per trarre definizioni e punti di riferimento che lo aiutino a orientarsi nel dibattito pubblico e politico. Questi ultimi sono invasi da questioni, immagini e definizioni tratte dal passato e, come ricordava Marc Bloch, «i nostri uomini d'azione hanno continuamente sulle labbra i suoi insegnamenti, veri o presunti» ${ }^{4}$. Allo stesso tempo, il passato ha dimostrato di poter offrire interessanti opportunità di commercializzazione, prontamente sfruttate dall'industria culturale per soddisfare i gusti di un pubblico che, a partire dagli anni Settanta del secolo scorso ${ }^{5}$, si è dimostrato sempre più incline a intrattenersi con la storia ${ }^{6}$. Tuttavia se volessimo confrontare la diffusione di prodotti legati alla storia con il riconoscimento tributato alla sua utilità sociale, ci troveremmo davanti al paradosso di un sapere ampiamente consumato ma che gode di uno scarso prestigio pubblico.

Tale contraddizione si ripercuote inoltre su chi della storia ha fatto il proprio mestiere e che, al di fuori della realtà accademica, stenta a far sentire la propria voce. La difficoltà di ottenere riconoscimenti in settori diversi dal proprio campo scientifico ha indotto molti storici a destinare «la totalità del proprio tempo al dibattito specialistico e autoreferenziale piuttosto che al pubblico più vasto e men che meno al condizionamento della politica» ${ }^{7}$. Il ripiegamento della storia accademica su questioni accessibili solo ai cultori della materia, unito alla crescente popolarità dell'economia intesa come una sorta di scienza oggettiva di governo, avrebbe provocato una frattura con colei che della storia dovrebbe essere interlocutrice privilegiata: la realtà politica.

Riflettendo sulla situazione della storia nel nostro tempo e sul suo legame con il potere diverse personalità della storiografia angloamericana hanno tuttavia denunciato la separazione tra la ricerca storica e la prassi di governo come artificiosa poiché, se è vero che la figura professionale dello storico può essere tenuta ai margini del dibattito politico, il passato influenza e continuerà a influenzare la politica e i suoi orientamenti ${ }^{8}$. L'idea che quello tra storia e politica e, più in generale, quello tra storia e vita pubblica, sia un dialogo che non si può spezzare costituisce uno

\footnotetext{
${ }^{4}$ BLOCH, Marc, Apologia della storia. O Mestiere di storico, Torino, Einaudi, 2009, p. 8.

${ }^{5}$ ROUSSO, Henry, «Applied History or the Historian as a Miracle-Worker», in The Public Historian, 6, 4/1984, pp. $65-85$, p. 68.

${ }^{6} \mathrm{Si}$ veda a titolo d'esempio: HIGGINS, Charlotte, «Tudormania: why can't we get over it?», in The Guardian, 4 maggio 2016, URL: < https://www.theguardian.com/news/2016/may/04/tudormania-why-can-we-not-getover-it > [consultato il 3 novembre 2017].

${ }^{7}$ BERNARDINI, Giovanni, «Una storia che serva alla politica (senza esserne serva)», in Mente Politica, 23 dicembre 2014, URL: < http://www.mentepolitica.it/articolo/una-storia-che-serva-alla-politica-senzaesserne-serva/310 > [consultato il 3 novembre 2017].

${ }^{8}$ RECORD, Jeffrey, Perils of Reasoning by Historical Analogy. Munich, Vietnam and American use of Force since 1945, Maxwell Air Force Base, Center for Strategy and Technology Air War College, 1998, p. 23.
} 
degli assunti principali della applied history la quale, di recente, è tornata a guadagnarsi l'attenzione degli specialisti come pure dell'opinione pubblica. I principi e gli obiettivi della applied history costituiscono il focus di questo articolo, così come l'analisi dei casi di studio costituiti dal progetto History \& Policy e dall'attività di ricerca svolta all'interno dell'Applied History Project del Belfer Center for Science and International Affairs. Tuttavia dal momento che la applied history può essere considerata un'evoluzione se non addirittura, come sostengono alcuni, una vera e propria appendice o specializzazione dell'indirizzo noto come public history, la prima parte dell'articolo consisterà in una panoramica sugli sviluppi della public history. D'altra parte, i due indirizzi si contendono un campo d'azione simile e sono stati oggetto delle stesse critiche e perplessità, per cui parlare dell'uno senza menzionare l'altro finirebbe con l'offrire una lettura parziale di entrambi.

Public history e applied history sono indirizzi di ricerca nati negli Stati Uniti e poi esportati in Europa, dove sono stati accolti con maggior entusiasmo da Paesi come Gran Bretagna e Paesi Bassi ${ }^{9}$. Di recente sono state le comunità scientifiche americane e britanniche ad aver rilanciato la riflessione sul legame tra storia e prassi politica. Di conseguenza, anche se non tutti gli autori citati possiedono un passaporto americano o britannico, la maggior parte delle fonti e le considerazioni da esse derivate sono tratte da lavori in lingua inglese e fanno riferimento al dibattito che si è sviluppato principalmente all'interno di università e centri di ricerca angloamericani.

\footnotetext{
${ }^{9}$ WESLEY JOHNSON, George, «An American Impression of Public History in Europe», in The Public Historian, 6, 4/1984, pp. 86-97. Per quanto riguarda l'Italia negli ultimi anni si è registrato un interesse crescente verso le questioni e le opportunità collegate alla public history. Una testimonianza di tale fenomeno sono le iniziative sempre più numerose dedicate alla materia tra cui spicca la prima conferenza nazionale sulla public history che si è svolta a Ravenna nel giugno 2017. Cfr. ASSOCIAZIONE ITALIANA DI STORIA ORALE, Prima conferenza nazionale di public history, URL: < http://aisoitalia.org/?p=4961 > [consultato il 23 marzo 2017]. A livello locale, inoltre, singoli atenei hanno iniziato a organizzare incontri e corsi di specializzazione post-laurea incentrati sulla public history. Cfr. ad es. il seminario "Public historians: gli storici tra accademia e mercato", URL: <https://seminariopermanentestoria.com/2017/03/02/24032017-_-public-historians-glistorici-tra-accademia-e-mercato-_/ > [consultato il 3 novembre 2017]; il master dell'Università di Modena e Reggio Emilia, URL: < http://www.masterpublichistory.unimore.it/site/home.html > [consultato il 4 novembre 2017].
} 


\section{Public History: la storia tra pubblico e mercato}

Nell'avvicinarsi allo studio della public history ci si imbatte subito nel problema della sua definizione. Nel 2008 il National Council on Public History (NCPH), l'organizzazione americana di riferimento per lo studio e le iniziative legate alla storia pubblica, presentò i risultati di un'indagine volta ad analizzare i principali settori professionali nei quali erano impiegati i public historians. I dati riportati nella figura 1 dimostrano che, all'infuori del tradizionale settore accademico e scolastico, la maggior parte degli storici intervistati ricopriva incarichi all'interno di istituzioni culturali quali musei e associazioni storiche, mentre una percentuale non indifferente lavorava per conto del governo (circa il $20 \%$ se si sommano le percentuali degli storici rispettivamente impiegati presso il governo federale e i governi degli Stati). Lo studio riportava un altro dato interessante, ovvero il fatto che molti intervistati rifiutavano di riconoscersi nella categoria di public historians:

Of the 3,856 people who responded to the survey, just 2,946 were willing to identify themselves as public historians - the remainder expressed some uncertainty about the term and whether it applied to them. In fact, 364 of the respondents who appeared to be employed as public historians (with long-term or professional positions in history outside of academia) declined to accept the label [...] Some found it too confining. As one observed, «A historian is a historian whether working in government, academia, or private industry» ${ }^{10}$.

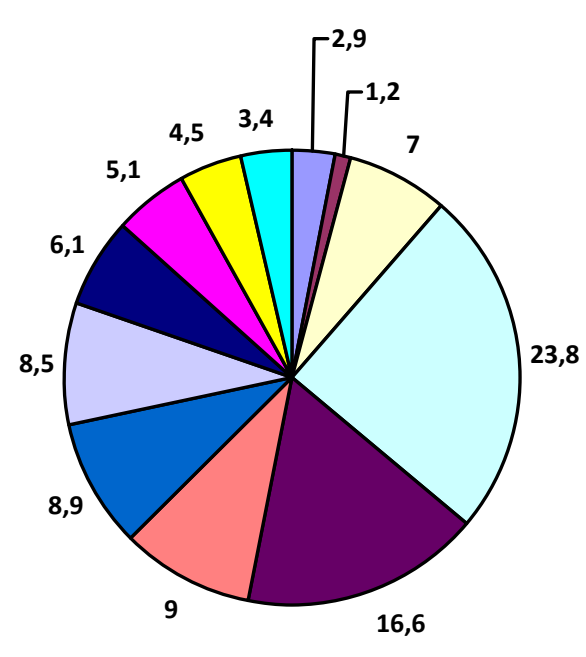

$\square$ Settore commerciale/industriale

Scuola primaria e secondaria

口Altro

$\square$ Sistema museale

College e università

Enti governativi e provinciali

$\square$ Giunte storiche

$\square$ Governo federale

Liberi professionisti

$\square$ Centri di ricerca/archivi/biblioteche

口Organizzazioni non-profit

$\square$ Società di consulenza

Figura 1. Settore di impiego dei public historians negli Stati Uniti. Elaborazione da un grafico del National Council on Public History.

\footnotetext{
${ }^{10}$ DICHTL, John, TOWNSEND, Robert, «A Picture of Public History. Preliminary Results from the 2008 Survey of Public History», in Public History News, 29, 4/2009, p. 1.
} 
Apparentemente, purtroppo, l'NCPH non ha raccolto dati più recenti circa l'impiego degli storici nei settori extra-accademici né ha condotto interviste analoghe su quanti, tra gli intervistati, si identifichino nella definizione di public historian. Tuttavia, ancora oggi, la public history sembra sottrarsi a facili identificazioni. Come ha notato Serge Noiret, il tentativo di racchiuderla in una definizione univoca, standardizzata, è divenuto un compito ancora più arduo da che la materia ha iniziato a diffondersi a livello internazionale. Assorbita da tradizioni storiografiche differenti, la public history si presenta come un percorso di ricerca in continua progressione ${ }^{11}$. Ma a fronte di una definizione labile le ragioni che hanno portato all'elaborazione della public history sono piuttosto chiare e vanno ricercate in primo luogo in quella che potremmo definire l'accentuazione o la formalizzazione di una tendenza intrinseca alla ricerca storica, ovvero quella di essere un «processo sociale» condiviso e orientato alla comunicazione dei propri risultati $^{12}$. In secondo luogo la sua comparsa può essere ricondotta alla necessità avvertita dagli storici di un «rinnovamento professionale» come risposta a due ordini di problemi: la riduzione delle opportunità di trovare un impiego all'interno del mondo universitario e la comparsa di un pubblico non specializzato affamato di storia ${ }^{13}$. Ma con il crescere dell'interesse generalizzato per la storia si sono moltiplicate le forme di trasmissione «agnostica» della stessa, le quali hanno lentamente ridefinito gli equilibri di forza tra storici e testimoni in favore dei secondi ${ }^{14}$. Si è così affermato una sorta di privilegio dell'osservatore in virtù del quale il significato della storia rivive non nei lavori di ricerca ma nella memoria di chi la storia l'ha vissuta ${ }^{15}$.

Tra il 1976 e il 1978 alcune facoltà di storia americane (in particolare l'University of California di Santa Barbara) iniziarono a riflettere su come arginare gli effetti della cosiddetta job crisis (cfr.

11 NOIRET, Serge, «Internationalizing Public History», in Public History Weekly, 2, 34/2014, URL: < https://public-history-weekly.degruyter.com/2-2014-34/internationalizing-public-history/ > [consultato il 4 novembre 2017].

${ }^{12}$ KALELA, Jorma, «History Making: The Historian as Consultant», in Public History Review, 20, 2013, pp. 2441. Di per sé la public history non presenta delle grandi differenze metodologiche rispetto alla storia tradizionale. Così si pronuncia l'NCPH in merito alla questione: «in terms of intellectual approach, the theory and methodology of public history remain firmly in the discipline of history, and all good public history rests on sound scholarship». Ciò che risulta davvero innovativo nell'approccio suggerito dalla public history è piuttosto l'attenzione posta ai rapporti e alle attività di collaborazione e networking con realtà istituzionali e imprenditoriali all'infuori del mondo accademico. Cfr. NATIONAL COUNCIL ON PUBLIC HISTORY, How is Public History different from "regular" history?, URL: < http://ncph.org/what-is-publichistory/about-the-field/ > [consultato il 4 novembre 2017].

${ }^{13}$ RAVVEDUTO, Marcello, «Una Italian Public History per la Seconda Repubblica», in Officina della Storia, 10, 2013, URL:

<http://www.officinadellastoria.info/magazine/index.php?option=com_content\&view=article\&id=334:unaitalian-public-history-per-la-seconda-repubblica\&catid=69:cantieri-sissco-2013\&Itemid=96 > [consultato il 7 novembre 2017].

${ }^{14}$ BENIGNO, Francesco, Parole nel tempo. Un lessico per pensare la storia, Roma, Viella, 2013, p. 17.

${ }^{15}$ FINK, Nadine, "Competition: The Power of Contemporary Witnesses», in Public History Weekly, 5, 5/2017, URL: < https://public-history-weekly.degruyter.com/5-2017-5/competition-the-power-of-contemporarywitnesses/ > [consultato il 7 novembre 2017]. 
figura 2), derivata dal fatto che le università formavano più dottorandi in storia di quanti ne potessero assorbire una volta conclusa il percorso di dottorato ${ }^{16}$.

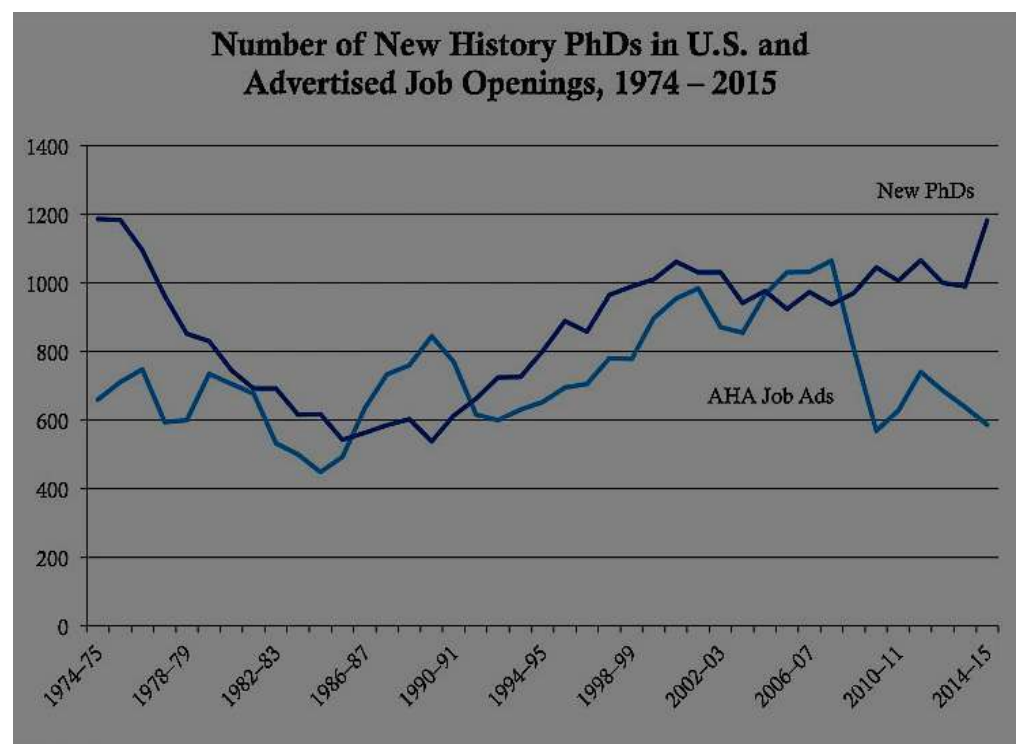

Figura 2. Numero di nuovi dottori di ricerca in Storia negli Stati Uniti e corrispettive posizioni di lavoro aperte.

Fonte: American Historical Association.

L'esigenza di trovare carriere alternative per i laureati in storia innescò una riflessione profonda sulle abilità intrinseche alla formazione storica (quali, ad esempio, capacità analitiche, di sintesi e abilità di problem solving) e sulla possibilità di impiegarle in realtà professionali, pubbliche o private, diverse dal mondo della ricerca. Furono così fondati alcuni corsi pensati per "allenare i giovani studiosi ad un approccio pragmatico all'analisi storica ${ }^{17}$. Fin da subito, i teorizzatori della public history misero in guardia dal considerare quest'ultima come una sorta di discorso inferiore, una mera strategia d'occupazione per gli storici, e sottolinearono invece come si trattasse di un'area disciplinare dotata di una propria dignità scientifica. Secondo Henry Rousso sarebbe perfino riduttivo voler individuare nella crisi occupazionale degli anni Settanta la causa principale della comparsa public history:

\footnotetext{
${ }^{16}$ WESLEY JOHNSON, George, "The Origins of "The Public Historian" and the National Council on Public History», in The Public Historian, 21, 3/1999, pp. 167-179; JONES, Arnita, «Public History Now and Then», in The Public Historian, 21, 3/1999, pp. 21-28.

${ }^{17}$ WESLEY JOHNSON, George, op. cit., p. 168. Uno di questi corsi fu fondato da Ernest May il quale ebbe un ruolo importante nel determinare gli indirizzi della public history e, come vedremo, della applied history. Nel 1978 May co-diresse un programma destinato agli studenti delle graduate schools, umanisti inclusi, denominato Careers in Business, basato sulla convinzione che «the relationship between academia and business is a potentially a fruitful one». Il programma era strutturato per fornire agli studenti delle nozioni basilari di management. Cfr. RATNER, Jonathan D., «Program to Ready Ph.D.s for Careers in Business», in The Harvard Crimson, URL: < http://www.thecrimson.com/article/1977/11/4/program-to-ready-phds-forcareers/ > [consultato il 7 novembre 2017].
} 
More than simply an original experiment, it is the locus of a historiographic revival affecting the entire discipline [...] Public history has brought about a renewal of interest in certain themes, and studies about government, the structures of economic decision-making and urban or industrial policies have multiplied [...] Public history and applied history are not creatures of the job crisis [...] they are an approach to using history in regard to contemporary society that has a valid intellectual base and that would have arisen [...] whether there was a job crisis or not ${ }^{18}$.

La public history ambisce ad esportare il metodo storico al di fuori delle università nella convinzione che la conoscenza del passato e le tecniche attraverso cui essa viene acquisita, costituiscano un mezzo per una migliore comprensione dei processi sociali e un'occasione di arricchimento per il dibattito pubblico ${ }^{19}$. Prima ancora che alla mancanza di opportunità lavorative la public history intende rispondere all'esigenza di comunicare il passato in una società nella quale lo sviluppo tecnologico ha radicalmente influenzato l'idea di passato e memoria e diversificato le possibilità di una loro trasmissione a un pubblico di non esperti ${ }^{20}$.

Un esempio delle opportunità che i public historian possono cogliere in un dibattito pubblico globalizzato spesso dominato dai temi dell'identità e della comunità nazionale, è fornito dalla controversia sulle memorie e i simboli della Guerra civile che si è riacutizzato negli Stati Uniti in seguito al massacro di Charleston (2015) e alla manifestazione dei suprematisti bianchi di Charlottesville (2017). L'esplosione di episodi di violenza razziale nel Sud degli Stati Uniti è stata accompagnata da un'accesa disputa sull'opportunità di preservare l'esposizione di simboli legati agli Stati Confederati d'America, fossero essi la bandiera confederata o le statue del generale Robert E. Lee $^{21}$.

I social network hanno naturalmente permesso che il dibattito pubblico fosse ampliato e amplificato dai commenti di osservatori non specializzati divisi tra coloro a favore della

\footnotetext{
${ }^{18}$ ROUSSO, Henry, op. cit., pp. 72-73.

${ }^{19}$ KELLEY, Robert, «Public History: Its Origins, Nature, and Prospects», in The Public Historian, 1, 1/1978, pp. 16-28.

${ }^{20}$ LIPSITZ, George, The case for studying popular culture, in FRANZ, Kathleen, SMULYAN, Susan (eds.), Major Problems in American Popular Culture, Boston, Wadsworth Publishing, 2011, p. 4. Rispetto al periodo in cui operavano i pionieri della public history, oggi la diffusione di ricerche che si sottraggono alle regole del discorso storico professionale è un problema reso ben più acuto dalla presenza dei social media e delle piattaforme free content. Tali siti hanno dissolto la divisione dei ruoli tra autore e lettore, consentendo a quest'ultimo di farsi a sua volta produttore e divulgatore di conoscenza storica, per altro senza doversi sottoporre agli stessi criteri di responsabilità e identificabilità cui invece sono tenuti gli storici di professione. Cfr. BALDO, Tommaso, «Riflessioni sulla narrazione storica nelle voci di Wikipedia», in Diacronie. Studi di Storia contemporanea, 29, 1/2017, URL:

< http://www.studistorici.com/2017/03/29/baldo_numero_29/ > [consultato il 7 novembre 2017].

${ }^{21}$ FELTON, Lena, HOSKING, Taylor, «The Legacy of Confederate Symbols», in The Atlantic, 17 agosto 2017, URL:

<https://www.theatlantic.com/politics/archive/2017/08/charlottesville-confederate-monuments/537177/> [consultato il 9 novembre 2017].
} 
rimozione-distruzione dei simboli confederati e coloro a favore del loro mantenimento. Un fronte, quest'ultimo, particolarmente variegato nel quale è possibile rintracciare tanto coloro che identificano nei monumenti una «testimonianza storica e non un simbolo d'odio» ${ }^{22}$, quanto i promulgatori di un'immagine edulcorata degli Stati del Sud afferente ai filoni narrativi della «Lost Cause» e del «Moonlight and Magnolia», fino ad arrivare ai suprematisti bianchi veri e propri. In tale contesto gli storici hanno saputo conquistarsi un ruolo di primo piano all'interno del dibattito utilizzando i canali di comunicazione online, sfruttandone la malleabilità e la capacità di raggiungere vaste platee ma allo stesso tempo piegandoli alla precisione metodologica della ricerca storica. L'interazione tra storici e pubblico non specializzato sui social network ha avuto esiti interessanti, come dimostra il "Charleston Syllabus», un progetto nato e finanziato attraverso Twitter volto a raccogliere documentazione relativa alla storia della schiavitù e del razzismo negli Stati Uniti, poi ordinato e trasposto in un'edizione cartacea ${ }^{23}$.

L'interesse suscitato da tali progetti di comunicazione storica, realizzati da professionisti ma divulgati attraverso media che possono raggiungere la cittadinanza nel suo complesso, suggerisce la possibilità di un rapporto comunicativo e formativo tra gli storici e la collettività, sia nazionale che internazionale, capace di svilupparsi non solo entro i confini immateriali del web ma anche e soprattutto all'interno delle comunità. La storia, caratterizzata da una dimensione educativa e civica molto profonda, si presta ad essere un utile strumento di lavoro al servizio della community engaged scholarship, un indirizzo incentrato sul dialogo tra società e accademia e sulla concezione della ricerca non come pratica isolata bensì come un processo integrato nella comunità, nella sua dialettica e nelle sue problematiche ${ }^{24}$.

Come ha osservato la storica statunitense Karen Cox, nel commentare il cospicuo numero di richieste ricevute da cittadini interessati ad approfondire gli aspetti più controversi della storia nazionale in seguito all'ultimo scontro sulle statue sudiste, la congiuntura storica sembrerebbe propizia per riaffermare il ruolo e la responsabilità pubblica della storia:

\footnotetext{
${ }^{22}$ Lo slogan heritage, not hate fu coniato negli anni Novanta da Charles Lunsford, membro del gruppo Sons of Confederate Veterans, per protestare contro il tentativo di rimuovere l'emblema della Confederazione dalla bandiera dello Stato della Georgia. Cfr. SMOTHERS, Roland, «South's Emblem to be retained on Georgia flag», in The New York Times, 10 marzo 1993, URL:

<http://www.nytimes.com/1993/03/10/us/south-s-emblem-to-be-retained-on-georgia-flag.html> [consultato il 9 novembre 2017].

${ }^{23}$ WILLIAMS, Chad, WILLIAMS, Kidada, BLAIN, Keisha (eds.), Charleston Syllabus: Readings on Race, Racism and Racial Violence, Athens, University of Georgia Press, 2016.

${ }^{24}$ Per un'analisi del concetto di community-engaged scholarship si veda: BOYER, Ernest, «The Scholarship of Engagement», in Bullettin of the American Academy of Arts and Sciences, 49, 7/1996, pp. 18-33.
} 
they [the people] want historical context for what happened in Charlottesville and other events happening in our nation and across the globe. In a word, they are hungry. And they are hungry for reliable history ${ }^{25}$.

Strettamente correlato all'obiettivo di costruire un ponte tra storia e vita pubblica, vi è quello di riaffermare il ruolo dello storico nella società contemporanea e di sottolineare quanto quella che lo studioso Eliot Cohen ha definito la «mente dello storico» possa essere applicata agli ambiti più diversi della realtà: dalla strategia militare ai processi di decision-making politici e imprenditoriali ${ }^{26}$. Questa nuova forma di storia, dunque, è pubblica in primo luogo perché riconosce come proprio interlocutore una vasta ed eterogenea platea di consumatori di storia che si estende ben oltre la comunità scientifica di riferimento; in secondo luogo perché afferma che l'ambito di applicazione della conoscenza storica, come pure l'ambito d'azione dello storico, dev'essere il dibattito civile ${ }^{27}$.

Uno dei meriti principali della public history è stato quello di ristabilire un rapporto diretto tra lo studio del passato e l'azione del presente e di riaffermare, in ultima analisi, che le radici della storia vanno ricercate nel «bisogno sociale di orientare la vita quotidiana attraverso il tempo» ${ }^{28}$. Una fondata conoscenza degli eventi passati è necessaria ai singoli osservatori per avere punti di riferimento all'interno del dibattito pubblico ma anche ai grandi attori sociali, istituzioni e aziende, che possono trovare nell'indagine storica e nelle sue procedure utili strumenti per migliorare le rispettive policy.

A ben vedere, il percorso iniziato negli Stati Uniti negli anni Settanta non ha soltanto riaffermato il carattere pragmatico e la valenza pubblica della disciplina storica, ma ha anche ispirato lo sviluppo di programmi di didattica della storia incentrati su un insegnamento dinamico della storia, intesa né come racconto né come memorizzazione di fatti, bensì come forma di ragionamento critico e analisi di fenomeni progressivi ${ }^{29}$. Tali percorsi formativi

\footnotetext{
${ }^{25}$ COX, Karen, «Historians need to use their power now», in CNN, 4 settembre 2017, URL:

< http://edition.cnn.com/2017/09/04/opinions/liberal-arts-degrees-not-useless-opinion-cox/index.html > [consultato il 9 novembre 2017].

${ }^{26}$ COHEN, Eliot, «The Historical Mind and Military Strategy», in Orbis, 49, 4/2005, pp. 575-588.

${ }^{27}$ NOIRET, Serge, «La "Public History": una disciplina fantasma?», in Memoria e Ricerca, 37, 2011, pp. 9-35.

${ }^{28}$ RÜSEN, Jörn, «The Didactics of History in West Germany: Towards a New Self-Awareness of Historical Studies", in History and Theory, 26, 3/1987, pp. 275-286, p. 276.

${ }^{29}$ Sono esempi in tal senso il programma Reading Like a Historian dello Stanford History Education Group, vero e proprio corso di educazione alla lettura e alla contestualizzazione delle fonti primarie. Uno degli obiettivi del corso è quello di stabilire, fin dal periodo della formazione scolastica, un contatto diretto tra gli studenti e i metodi dell'indagine storica. Il focus è posto perciò sul procedimento analitico anziché mnemonico: «instead of memorizing historical facts, students evaluate the trustworthiness of multiple perspectives on historical issues. They learn to make historical claims backed by documentary evidence». Cfr. Reading Like a Historian, URL: < https://sheg.stanford.edu/rlh > [consultato il 9 novembre 2017]. Il corso dedicato alla storia della democrazia americana tenuto da David Moss alla Harvard Business School propone una didattica della storia ancora più innovativa, basata sull'uso di casi di studio (aperto perciò alla
} 
condividono con la public history l'enfasi posta sullo studio del passato inteso quale prassi effettiva di ricerca orientata all'azione. Quest'ultima consente allo studente di sviluppare un metodo di indagine e una consapevolezza storica che altro non è se non l'abitudine a interpretare dati che provengono da luoghi ed epoche distanti dalla propria e ad esaminare «processi inaspettati e non lineari» ${ }^{30}$. Lo storico, insomma, possiede tutti gli strumenti per gestire l'ambiguità, quella che Stephen Jay Gould riteneva «l'elemento più prezioso di qualsiasi analisi adeguata». Come vedremo, sarà proprio la familiarità dello storico con ciò che è mutevole a costituire una delle argomentazioni più forti a favore di una rinnovata intesa tra storia e politica.

\section{Applied history: la storia al potere}

La public history ha cercato di espandere l'applicazione della storia a settori eterogenei del mercato non solo per promuovere un uso più consapevole dei riferimenti al passato ma anche per riconquistare agli storici e ai loro sforzi un certo prestigio. Ma giacché sulle sue conclusioni e i suoi metodi non esiste un accordo unanime siamo ben lontani dal considerare il rapporto tra storia e dimensione pubblica come privo di contrasti. L'idea che la storia debba essere impiegata per risolvere controversie attuali e che lo storico abbia l'interesse (o il dovere) ad unirsi allo scontro di opinioni nell'agone pubblico, non è da tutti condivisa, per la frustrazione di coloro che invece rimproverano la tendenza degli storici a rimanere ai margini della società. L'australiano Paul Ham, ad esempio, si è polemicamente interrogato sull'utilità di una ricerca storica autoreferenziale, concepita per essere divulgata esclusivamente all'interno del mondo accademico:

Academic history is produced on a sort of enclosed carousel, whose rider's chief role is to compose a brief analyses or micro-histories - finely hewn articles for scholarly journals that their peers are obliged to review as an occupational duty, and make you wonder: what is academic history for? [...] To be popular in certain ivory towers is the kiss of death ${ }^{31}$.

contaminazione con i metodi di insegnamento propri dell'economia) e sull'immedesimazione diretta degli studenti nello svolgersi dell'evento storico esaminato: «the case method goes beyond historical skills and factual content; it aims to hone decision-making skills [...] Unlike with many history courses, where students look back at historical events students in Moss's course play history forward. If you were in that place as that voter, that labor leader, or that congressperson, what decision would you have made?». GROSS-LOH, Christine, "A Better Way to Teach History», in The Atlantic, 8 febbraio 2016, URL:

< https://www.theatlantic.com/education/archive/2016/02/harvard-history-class/460314/ > [consultato il 9 novembre 2017].

${ }^{30}$ GAVIN, Francis, «Thinking Historically: a guide for strategy and statecraft», in War on the Rocks, 17 novembre 2016, URL: < https://warontherocks.com/2016/11/thinking-historically-a-guide-for-strategyand-statecraft/ > [consultato il 9 novembre 2017].

31 HAM, Paul, «Human factors», in The Age, 22 marzo 2014, URL: < http://www.theage.com.au/entertainment/books/human-factors-20140319-353nd > [consultato il 9 
D'altra parte, alcuni osservatori hanno ribadito che le ricerche settoriali e specialistiche costituiscono una parte irrinunciabile del lavoro di storico (aspetto riconosciuto tra l'altro dallo stesso Ham) e che non necessariamente sono sintomo di una concezione elitista della storia e della sua funzione. Inoltre, i temi di indagine dovrebbero essere scelti in base a criteri scientificamente più consistenti che non gli orientamenti del dibattito contemporaneo o le tendenze del pubblico ${ }^{32}$. Il contrasto tra accademia e agorà costituisce uno dei problemi principali sui quali la public history ha indotto a riflettere: gli storici che ambiscono ad essere soggetti pubblicamente rilevanti saranno in grado di dettare il passo del dibattito, di influenzarne i contenuti, oppure dovranno adeguarsi a studiare ciò che interessa l'audience (o il cliente, nel caso di uno storico coinvolto in una relazione contrattuale con un'azienda)? E se così non fosse, lo storico non rischierebbe di perdere la propria autonomia e di rinunciare alla propria etica professionale ${ }^{33}$ ? Si tratta di un dubbio ancora più forte qualora si prendano in esame i rapporti tra analisi storica e dibattito politico.

novembre 2017]. Il distacco dei ricercatori dal dibattito pubblico è avvertito con una particolare urgenza negli Stati Uniti dove recentemente le critiche alla cosiddetta «cultura dell'esclusività» (ovvero la tendenza degli studiosi a comunicare solo tra pari) sono state accompagnate dagli appelli per un maggior coinvolgimento degli scholars (storici ma anche scienziati) quale unico rimedio contro una tendenza antiintellettuale sempre più radicata nella cultura americana. Cfr. KRISTOFF, Nicholas, «Professors we need you!», in The New York Times, 15 febbraio 2014; LEPORE, Jill, «The New Economy of Letters», in The Chronicles of Higher Education, 3 settembre 2013, URL: < http://www.chronicle.com/article/The-New-Economy-ofLetters/141291/ > [consultato il 9 novembre 2017]. Sull'esistenza di una cultura anti-scientifica in America si veda: HOFSTADTER, Richard, Anti-intellectualism in American Life, Knopf, New York, 1966.

32 MICHAELS, Paula, "What is academic history for?», in The Conversation, 25 marzo 2014, URL: < https://theconversation.com/what-is-academic-history-for-24795 > [consultato il 9 novembre 2017].

${ }^{33}$ Come accennato, public history e applied history hanno sollevato alcune perplessità e la questione dell'autonomia dello storico desideroso di collocarsi sul mercato è una delle più dibattute. Dell'argomento si è occupato R. C. Tobey secondo cui i public historian dovrebbero dotarsi di un codice deontologico specifico. Lo storico "su committenza", impiegato presso un ente non di ricerca, si trova esposto a un conflitto di interesse al quale lo storico tradizionale non era abituato: «from the point of view of the public arena, there is no ground of absolute disinterestedness on which public scholar may stand. Other parties will perceive and respond to the public scholar as if that scholar represented an interest [...] consequently it is necessary for the public scholar to adopt a conflict of interest ethical statement that will satisfy the expectations of the public arena». TOBEY, Ronald, «The Public Historian as Advocate: Is Special Attention to Professional Ethics Necessary?», in The Public Historian, 8, 1/1986, pp. 21-30, p. 28. Ma se è vero che la nuova relazione tra storico e committente chiede al primo di ridefinire il proprio codice di condotta, ciò non implica che l'etica dello storico sia necessariamente più a rischio all'esterno dell'accademia che non al suo interno. Innanzitutto public history e applied history si basano sul medesimo rigore disciplinare della storia d'accademia. Inoltre anche gli ambienti di ricerca sono stati di recente investiti da controversie etiche, trattandosi di luoghi in cui l'elevata competizione induce numerosi ricercatori a subordinare l'accuratezza dei propri studi al bisogno immediato di ottenere nuove pubblicazioni. Sull'assenza di un controllo qualità efficace nel mondo universitario ha indagato il «The Economist» (benché con uno studio incentrato soprattutto sull'ambito della hard science). (fr. «Problems with Scientific Research. How science goes wrong», in The Economist, 21 ottobre 2013, url: < https://www.economist.com/news/leaders/21588069scientific-research-has-changed-world-now-it-needs-change-itself-how-science-goes-wrong $>$ [consultato il 30 novembre 2017]. 
Gli storici sono spesso restii all'idea di introdurre il proprio lavoro nelle aule parlamentari, memori «degli usi e degli abusi» commessi dalla politica ai danni della storia durante il Novecento $^{34}$. Ma tale diffidenza non può annullare il fatto che un rapporto diretto tra cittadini, istituzioni e storia si consuma tutti i giorni «senza la mediazione della disciplina accademica» ${ }^{35}$. Questo stato di cose ha permesso a persone manchevoli di una solida preparazione storica di ergersi a interpreti del delicato legame tra le società e il proprio passato e, quale diretta conseguenza, che la storia sia «manipolata a fini propagandistici e trasformata in strumento esplicito di lotta politica» ${ }^{36}$.

Al cuore della applied history vi è il desiderio di ribadire che la relazione tra storia e politica non è e non deve essere strumentale quanto piuttosto utilitaristica: la conoscenza del passato può aiutare a formare una cittadinanza consapevole e ad elaborare politiche lungimiranti. Tuttavia affinché tale conoscenza sia utile e - in quanto tale - scientificamente fondata, è necessario che gli storici non si sottraggano al confronto con le autorità politiche.

Nonostante la storiografia americana abbia sviluppato per prima l'idea di applied history, oggi quest'ultima viene considerata da alcuni come una declinazione della public history ${ }^{37}$. Peter Stearns, che si è occupato diffusamente dei rapporti tra storia e scienze sociali, ritiene che essa faccia «parte della public history nel suo significato più ampio», pur ponendosi un «obiettivo più specifico» ${ }^{38}$. Anche l'NCPH afferma che applied e public possono essere usati come sinonimi e, del resto, non va dimenticato che gli storici fondatori dell'NCPH pensarono in un primo momento di riferirsi alla disciplina che andavano teorizzando come applied history, termine poi abbandonato in favore del meno «aggressivo» public history ${ }^{39}$. Il legame tra applied history e public history sembra inoltre essere confermato dal fatto che entrambe iniziarono ad essere teorizzate nel medesimo periodo: il primo corso ufficiale di public history fu istituito nel 1976 dalla University of California a Santa Barbara, con il patrocinio della Rockefeller Foundation. Nello stesso anno comparve il primo percorso di specializzazione in storia applicata promosso dalla Carnegie-Mellon University di Pittsburgh. Il corso della Carnegie-Mellon si distingueva per l'impegno nell'integrare una

\footnotetext{
${ }^{34}$ GREEN, Alix, History, Policy and Public Purpose. Historians and Historical Thinking in Government, London, Palgrave MacMillan, 2016, p. 13.

${ }^{35}$ Ibidem, p. 14.

${ }^{36}$ VIDOTTO, Vittorio, Guida allo studio della storia contemporanea, Roma-Bari, Laterza, 2011, p. 16; cfr. GALLERANO, Nicola (a cura di), L'uso pubblico della storia, Franco Angeli, Milano, 1995.

${ }^{37}$ Risale al 1914 una delle prime opere in cui si menziona la applied history. Si tratta del pamphlet dell'americano Benjamin Shambaugh, il quale offre una definizione della disciplina che, al netto degli echi positivisti dai quali è percorsa, è decisamente affine alle sue più recenti rielaborazioni: «Applied History is simply the use of the creative power of scientific knowledge in politics and administration [...] Applied History is not a dream but a sound and intelligent method of interrogating the past in the light of the conditions of the present and the obvious needs of the immediate future». SHAMBAUGH, Benjamin, Applied History, Iowa City, Iowa State Historical Society, 1914, p. 8.

${ }^{38}$ STEARNS, Peter, «Applied History and Social Sciences», in Social Science History, 6, 2/1982, pp. 219-226, p. 219.

${ }^{39}$ ROUSSO, Henry, op. cit., p. 69.
} 
preparazione «interdisciplinare» con un approccio di ricerca e di lavoro saldamente radicato nel metodo storico ${ }^{40}$. Rispetto al corso di applied history che Ernest May e Richard Neustadt avrebbero fondato ad Harvard nel 1978, e che a sua volta sarebbe stato determinante per i futuri sviluppi della storia applicata, il programma della Carnegie rappresentava un percorso di formazione professionale pensato per gli storici, dagli storici. Mentre il corso di Harvard si proponeva di introdurre al metodo storico funzionari già al servizio degli enti governativi, quello della Carnegie si prefiggeva di fornire agli studenti di storia le conoscenze necessarie per ricoprire incarichi amministrativi e di governo ${ }^{41}$.

Concepite quasi in contemporanea e unite da un'affinità di metodi e intenzioni, applied history $\mathrm{e}$ public history costituiscono due approcci di ricerca correlati ma allo stesso tempo dotati di specifiche identità. Entrambe affrontano il legame tra discorso storico e scena pubblica e ribadiscono il carattere pragmatico della conoscenza storica. Ma nel quadro di pensiero generale elaborato da storici come John Tosh, Niall Ferguson e Graham Allison la applied history si distingue per la promozione di un approccio storico all'analisi politica e per aver riproposto in chiave originale la relazione tra storiografia e politica. Secondo l'applied history la storia non è solo un quadro interpretativo del passato ma è anche un orientamento per le strategie politiche presenti e future. Volendo adattare al nostro contesto una frase che Linneo pronunciò a proposito della natura, potremmo dire che historia non facit saltus. La relazione tra storia e politica è complicata e proteiforme ma tuttavia inestinguibile, giacché tutte le scelte politiche maturano all'interno di una cornice storica in cui il presente è connesso al passato in quanto da esso si origina.

Se le considerazioni della applied history si fermassero qui, avremmo a che fare con una disciplina ben poco innovativa. Il legame tra storia e politica non è stato certo trascurato dalle tradizioni storiografiche occidentali e l'idea che la storia sia maestra di vita è un topos tanto antico quanto abusato. L'applied history, tuttavia, non si limita ad appellarsi a una concezione didascalica del passato, si impegna invece a individuare i modi in cui l'educazione storica e, soprattutto, i processi analitici a cui lo storico è abituato, possono stimolare e arricchire i processi decisionali a livello governativo. La applied history cerca di realizzare, insomma, una costruzione storicamente fondata dell'agire politico, ritagliandosi così un ruolo particolare rispetto non solo alla storia pubblica ma anche rispetto alla storia contemporanea tradizionale. L'applied history propone una visione della storia a un tempo come accertamento critico dei fatti e come strumento per indagare nuove, possibili, forme d'azione. Il rifiuto di stabilire una divisione tra il momento teorico e il momento pratico dell'indagine costituisce l'istanza specifica della storia applicata. Peter Stearns ha suggerito di guardare a quest'ultima come a una disciplina ibrida, capace di combinare il

\footnotetext{
${ }^{40}$ STEARNS, Peter, TARR, Joel, «Applied History: A New-Old Departure», in The History Teacher, 14, 4/1981, pp. 517-531, p. 527.

${ }^{41}$ Ibidem, p. 518.
} 
metodo della ricerca storica con gli obiettivi delle scienze sociali, economia e scienze politiche in primis:

In a sense, applied historians are taking into the policy realm the larger interest in a new union between history and social sciences. Many applied historians, like their social science history counterparts, see their mission mainly in terms of fuller historical data to accomplish the basic purposes of social science: better theory, more precise methodology, and [...] a closer alignment to policy needs ${ }^{42}$.

L'applied historian si distinguerebbe inoltre dal contemporaneista sia per la selezione dei temi di ricerca (dettata dalle contingenze politiche e dalle esigenze delle istituzioni con cui l'applied historian collabora, ovvero da circostanze esterne al ricercatore), sia per la modalità di esposizione dei risultati dell'indagine, nella misura in cui il primo rinuncerebbe a un'analisi di tipo discorsivo:

One of the principal differences between applied history and most contemporary history, de facto, is the former's avoidance of a narrative mode. The applied historian is looking for elements in the past that will explicate, possibly guide, in a current problem situation. He is not content to provide an introductory back-ground statement which will locate and inform serious policy research but not be a part of $\mathrm{it}^{43}$.

Leggendo le elaborazioni teoriche della applied history si nota come esse cercano di assolvere due obiettivi precisi: evidenziare le affinità tra l'indagine storiografica e la sua controparte politica; esaminare l'armamentario analitico dello storico e valutare in che modo e fino a che punto esso possa essere d'aiuto ai politici e ai loro tecnici. Per quanto concerne il primo obiettivo, gli applied historian affermano una sostanziale omologia tra storia e politica, poiché «entrambe si originano da un problema che necessita di essere spiegato» ${ }^{44}$. La storia è una disciplina sintetica, procede attraverso lo studio di fonti eterogenee e dispone $\mathrm{i}$ dati raccolti in una cornice cronologica e interpretativa. La storia, al pari della politica, lavora sull'analisi e l'integrazione di informazioni. L'ordinamento dei dati non esaurisce però il compito della ricerca storica perché, se così fosse, essa si limiterebbe a descrivere uno scenario senza spiegarlo o interpretarlo. Rifuggendo una concezione descrittiva dell'indagine storica, i teorici della applied history hanno isolato tre strumenti propri dell'indagine storiografica che, a loro giudizio, sarebbero di massima importanza per l'analisi politica, ovvero: la periodizzazione, la contestualizzazione e l'analogia.

\footnotetext{
${ }^{42}$ STEARNS, Peter, op. cit., p. 224.

${ }^{43}$ Ibidem, p. 222.

${ }^{44}$ GREEN, Alexis, op. cit., p. 80.
} 
Periodizzazione e contestualizzazione sono procedimenti correlati tra di loro. Nel momento in cui si prende in esame un certo problema politico, la costruzione di un quadro cronologico è un'operazione imprescindibile per poter collocare quel dato problema in una corretta prospettiva storica. A sua volta la contestualizzazione si rivela necessaria sia per accertare un percorso evolutivo coerente della questione, sia per ipotizzare scenari alternativi:

By reassembling the historical building blocks of a policy issue [...] the historian enlarges the options for policy action in the present by showing the array of alternatives that were plausible and feasible in the past 45 .

La fase preliminare della contestualizzazione consente di articolare i fatti storici che hanno determinato l'insorgere di una situazione specifica all'interno di una sequenza unitaria e dunque di considerarli non momenti isolati, bensì eventi concatenati di un medesimo pattern ${ }^{46}$. Questa prospettiva organica sul passato implica, secondo gli applied historian, dei sicuri vantaggi interpretativi che le classi dirigenti potrebbero sfruttare per pianificare delle strategie a lungo termine. Formulare l'azione politica alla luce della prassi e delle conoscenze dell'indagine storiografica impedirebbe inoltre di perpetrare l'uso fallace di un dispositivo concettuale proprio dello storico ma tuttavia molto apprezzato dai politici: l'analogia.

L'uso appropriato dell'analogia è ciò che contraddistingue un impiego consapevole ed efficace del passato da una sua vuota attualizzazione. L'analogia è uno strumento con un forte potere espressivo e rappresenta la forma con cui, prevalentemente, la storia rivive nel dibattito politico. L'analogia condensa fenomeni del passato, momenti spartiacque della storia e li trasforma in sintesi simboliche: il piano Marshall, l'appeasement, il Vietnam, per citare alcune delle analogie più ricorrenti, diventano macro-categorie capaci di creare rimandi immediati tra passato e presente, sulla base dei quali le iniziative politiche vengono spiegate e legittimate.

L'impiego dell'analogia da parte di chi non è abituato ad operare professionalmente con i fatti storici presenta evidenti limiti ed è proprio in virtù di questo cattivo uso della comparazione con il passato che gli applied historian rivendicano la necessità di introdurre gli storici nei ministeri e negli uffici di governo. Sottrarre le analogie alle applicazioni imprecise di cui sono state spesso oggetto rappresenta, per la applied history, il primo passo verso la riqualificazione del ruolo della storia nel dibattito politico. La comparazione tra fenomeni politici e circostanze storiche differenti può rivelarsi fuorviante, se non dannosa, sotto il profilo strategico. Un esempio delle distorsioni provocate da un uso malaccorto dell'analogia viene fornito da May nel suo Lessons of

\footnotetext{
${ }^{45}$ Ibidem, p. 68.

${ }^{46}$ VERTZBERGER, Yaacov, «Foreign Policy Decisionmakers As Practical-Intuitive Historians: Applied History and Its Shortcomings», in International Studies Quarterly, 30, 2/1986, pp. 223-247, p. 227.
} 
the Past, in cui si sottolinea l'errore di valutazione commesso dall'amministrazione Truman nel ritenere che la situazione europea del secondo dopoguerra avrebbe ricalcato quella del 1919 e, nello specifico, nell'aver individuato una rispondenza tra l'espansionismo sovietico nei Balcani con quello della Germania nazista nell'Europa continentale ${ }^{47}$.

Se da un punto di vista pratico una analogia scentrata può incidere negativamente su una decisione politica, dal punto di vista teorico dello storico essa può tradire l'appropriazione ideologica e la trasfigurazione retorica del passato. È quanto accaduto, ad esempio, negli Stati Uniti dopo l'11 settembre, quando la retorica presidenziale attingeva al ricordo della Seconda guerra mondiale per descrivere ciò che stava avvenendo in Medio Oriente e suggeriva paragoni tra il conflitto iracheno e la guerra di liberazione in Europa ${ }^{48}$. Ma a supportare l'insistenza degli applied historian su un uso fondato della comparazione storica potrebbero essere citati numerosi casi, tra cui quello del classicista Alexander Demandt, autore di un controverso articolo pubblicato sulla «Frankfurter Allgemeine Zeitung»e incentrato sulla similitudine tra le invasioni barbariche, il crollo dell'Impero romano e l'attuale crisi dei migranti. L'articolo di Demandt, nel quale la spiegazione semplificata delle ragioni che scatenarono la crisi dell'impero si somma all'impiego di una terminologia contemporanea impropria - l'uso del termine rifugiati per definire i Goti ne è un esempio palese - costituisce solo uno degli ultimi esempi di un impiego «banalizzato, problematico e [...] anacronistico» della storia nel discorso politico ${ }^{49}$. Nei casi succitati l'analogia non viene usata come strumento di interpretazione attiva del passato e del presente. Di conseguenza la storia si ritrova a svolgere una funzione apologetica, di legittimazione dell'operato dell'autorità e di semplice convalida del punto di vista dell'interprete.

Nel 2003, davanti al Congresso americano, Tony Blair affermò che lo studio della storia aveva ormai ben poco da insegnare per la comprensione dei tempi presenti. La dichiarazione sembrò da un lato porre fine al legame consolidato tra la storia e la politica britannica ${ }^{50} \mathrm{e}$, dall'altro, fornì una chiara manifestazione di quella prigione concettuale che gli storici Jo Guldi e David Armitage ribattezzarono, anni dopo, come short termism, ovvero la tendenza delle analisi politiche come pure delle dissertazioni accademiche ad appiattirsi su orizzonti temporali di breve periodo ${ }^{51}$. Il manifesto di Guldi e Armitage sposa diversi punti programmatici della applied history, soprattutto nella misura in cui ritiene che la storia debba tornare ad essere considerata il «luogo di

\footnotetext{
${ }^{47}$ MAY, Ernest, Lessons of the Past. The Uses and Misues of History in American Foreign Policy, Oxford, Oxford University Press, 1975.

${ }^{48}$ NOON, David, «Operation Enduring Analogy: World War II, the War on Terror, and the Uses of Historical Memory», in Rhetoric \& Public Affairs, 7, 3/2004, pp. 339-364.

${ }^{49}$ THÜNEMAN, Holger, «Historia Magistra Vitae? The Banality of Easy Answers», in Public History Weekly, 3, 4/2016, URL: < https://public-history-weekly.degruyter.com/4-2016-3/historia-magistra-vitae-banalityeasy-answers/ > [consultato il 10 novembre 2017].

${ }^{50}$ ANDERSON, Olive, «The Political Uses of History in Mid Nineteenth-century England», in Past \& Present, 36, 1967, pp. 87-105.

${ }^{51}$ GULDI, Jo, ARMITAGE, David, The History Manifesto, Cambridge, Cambridge University Press, 2014.
} 
formazione della politica» ${ }^{52}$ e difende l'idea di uno storico immerso nel mondo, un «interprete qualificato della società d'oggi che non rifugge le sfide dell'attualità ${ }^{53}$. Ma per la storiografia britannica il testo di Guldi e Armitage non rappresenta la prima sortita nel panorama della storia applicata.

Fondato nel 2002 dietro l'iniziativa di un gruppo di storici affiliati a Cambridge e al King's College di Londra, il network History \& Policy si propone di «promuovere una migliore politica pubblica attraverso una maggiore comprensione della storia» ${ }^{54}$. Lo scopo delle iniziative di History \& Policy è quello di svolgere un processo di selezione dei dati storici al fine di integrarli nella formulazione delle politiche governative. L'impostazione originale di History \& Policy risiede nella convinzione che storia e politica si intersechino ad ogni livello di governo: rispetto ai percorsi di ricerca dell'Applied History Project che, come vedremo, prediligono esplorare i legami più convenzionali tra indagine storica e politica estera, History \& Policy ritiene che l'agire politico dovrebbe enucleare in sé una forte consapevolezza storica anche per ciò che riguarda le politiche sanitarie, di genere e di gestione della pubblica amministrazione. Il voler delineare un percorso di elaborazione politica che complessivamente, sotto i suoi diversi aspetti, riposi sulla conoscenza del passato rappresenta un'istanza per altro sempre più dibattuta nell'establishment di governo britannico: diversi incontri, tenutisi alla presenza di parlamentari e funzionari civili hanno perorato la causa di introdurre un consulente storico nei dipartimenti di Whitehall. Promotori di questa iniziativa sono stati, tra gli altri, il professor David Cannadine e Lord Butler, ex direttore dell'Home Civil Service ${ }^{55}$. Secondo Cannadine:

Historians and politicians bring very different perspectives to bear on the contemporary world and greater dialogue between them would be beneficial to the policy process. Historians can suggest, on the basis of past precedents, what might or might not work and counsel against raising public expectations that policies will be instantly effective. This would be particularly valuable in policy areas such as constitutional reform, which have a long and complex history that must be understood to make the right decision today ${ }^{56}$.

\footnotetext{
${ }^{52}$ IID., «The Bonfire of the Humanities», in Aeon, URL: < https://aeon.co/essays/the-role-of-history-in-asociety-afflicted-by-short-termism $>$ [consultato il 10 novembre 2017].

${ }^{53}$ NOIRET, Serge, DELAFONTAINE, Ramses, VERREYCKEN, Quentin, ARNESEN, Eric, «The History Manifesto: a discussion", in Memoria e ricerca, 51, 1/2016, pp. 97-126.

${ }^{54}$ History \& Policy, URL: < http://www.historyandpolicy.org/who-we-are > [consultato il 10 novembre 2017].

${ }^{55}$ «Every department should have a historical adviser, argues Lord Butler of Rockwell», in Civil Service World, 13 marzo 2013, URL: < http://www.civilserviceworld.com/every-department-should-have-a-historicaladviser-argues-lord-butler-of-brockwell/ > [consultato il 10 novembre 2017]; TENEMBAUM, Yoav, «The case for historical advisers in government», in History \& Policy, 10 ottobre 2009, URL:

$<$ http://www.historyandpolicy.org/opinion-articles/articles/the-case-for-historical-advisers-ingovernment> [consultato il 14 novembre 2017].

56 «Call for Government to Appoint Chief Historical Advisers», in History \& Policy, URL: < http://www.historyandpolicy.org/docs/chief_historical_adviser_release.pdf > [consultato il 14 novembre 2017].
} 
Il cuore di History \& Policy è il portale online dove sono raccolti i progetti di ricerca, i cui temi sono inseriti all'interno di un confronto serrato con l'attualità politica britannica. La maggior parte dei lavori ricorre a un ragionamento per analogie intenzionato a rilevare tanto la continuità quanto i cambiamenti con il passato. I membri di History \& Policy, dunque, rifuggono dall'utilizzare l'analogia come strumento per un'assimilazione forzata del passato e riconoscono che lo sforzo d'analisi dello storico non si limita a individuare buone analogie ma anche a smascherare quelle inesatte ${ }^{57}$. Uno dei progetti attualmente in corso si intitola Imagining Markets e analizza come, dal 1900 ad oggi, sono stati concepiti il ruolo della Gran Bretagna nel commercio mondiale e i suoi rapporti con l'Europa, il Commonwealth e la Cina. Ad oggi, dopo l'avvio della Brexit, la posizione della Gran Bretagna nel mercato internazionale figura su tutte le testate nazionali e internazionali ma, lungi dal considerarla una terrificante novità, gli autori associati al progetto ricordano che «la ridefinizione degli orientamenti economici della Gran Bretagna»su scala mondiale sono in realtà una costante della storia nazionale e che, perciò, è necessario sviluppare una visione organica dello sviluppo storico-economico del Paese qualora si voglia ampliare davvero la dimensione di lettura delle sfide e dei rischi posti dalla Brexit.

Al fine di diffondere un'interpretazione storicamente aggiornata della politica contemporanea, History \& Policy organizza e coordina seminari e workshop di ricerca in collaborazione con i dipartimenti pubblici, a dimostrazione della volontà di stabilire una comunicazione diretta con il mondo delle istituzioni. Secondo John Tosh, i membri di History \& Policy riescono a conciliare la figura dello storico con quella del consigliere politico senza venir meno al rispetto delle «norme accademiche ${ }^{58}$. Ma se il rigore nella preparazione dell'indagine è quello dello storico da accademia, lo stile e la presentazione sono esempi brillanti di una letteratura di informazione pensata per rendere immediatamente accessibili i propri contenuti a un pubblico (formato in prevalenza da funzionari di governo) che necessita di metabolizzare in fretta le notizie. La sintesi del processo di trasmissione dell'analisi storica cerca così di conciliarsi con la velocità tipica dei processi di decision-making ${ }^{59}$.

L'Applied History Project (AHP) del Belfer Center for Science and International Affairs, associato alla Harvard Kennedy School, può essere considerato la controparte americana di History \& Policy, dal momento che si prefigge scopi e procedure affini al gruppo britannico. L'intento del progetto è quello di raccogliere materiale che possa essere usato da «storici di

\footnotetext{
${ }^{57}$ TOSH, John, «In Defence of applied history: the History \& Policy website», in History \& Policy, 10 febbraio 2006, URL: < http://www.historyandpolicy.org/policy-papers/papers/in-defence-of-applied-history-thehistory-and-policy-website > [consultato il 14 novembre 2017].

${ }^{58}$ TOSH, John, «In Defence of applied history: the History \& Policy website», cit.

${ }^{59}$ REYNOLDS, David, «The Return of Big History», in The Newstatesman, 29 gennaio 2015, URL:

< http://www.newstatesman.com/politics/2015/01/return-big-history-long-past-antidote-short-termism > [consultato il 14 novembre 2017].
} 
professione, analisti e da chiunque sia interessato alla applied history ${ }^{60}$. Rispetto all'iniziativa britannica l'AHP si dimostra più flessibile e incline a una maggior interazione con un'utenza non specializzata. Se per collaborare con History \& Policy è richiesta una precisa affiliazione accademica o istituzionale, l'AHP si dimostra invece pronto ad accogliere suggerimenti, proposte di casi di studio e di assignment da sottoporre all'attenzione dei propri ricercatori. Figure di riferimento all'interno del progetto sono Niall Ferguson e Graham Allison, i quali cercano di raccogliere l'eredità di quegli storici americani (da Schlesinger Jr. a Kissinger fino ai citati May e Neustadt) che per primi hanno stabilito un legame profondo tra storiografia e azione di governo.

Il focus delle ricerche dell'AHP è certamente rappresentato dalle questioni relative alla politica estera americana. La predilezione per un'analisi storica che si concentri in modo prioritario sugli sviluppi e le crisi dello scenario geopolitico è del resto rispecchiata nell'iconico manifesto firmato da Ferguson e Graham, il quale propone di creare un consiglio di storici da affiancare al presidente degli Stati Uniti ${ }^{61}$. Ferguson e Graham si scagliano contro l'amnesia storica che affligge l'establishment americano e, a ben vedere, l'history deficit di cui parlano assomiglia a un analogo, o a un riflesso, dello short termism criticato da Guldi e Armitage. Ma, rispetto a questi ultimi, Ferguson e Graham adottano un'argomentazione più propositiva, elencando una serie di incarichi che il presidente potrebbe assegnare al suo consiglio di storici. I temi di indagine suggeriti dal testo si concentrano in particolare sui rischi fronteggiati dagli USA in regioni delicate come il Medio Oriente e il Sud-Est asiatico ${ }^{62}$. Così, ad esempio, in relazione alla drammatica ascesa dello Stato Islamico, i due storici consigliano di valutare anzitutto l'essenziale novità del fenomeno, vagliando con attenzione diversi gruppi estremisti, non necessariamente legati all'Islam radicale, per scovare precedenti simili ed evitare paragoni immediati ma non per forza adeguati:

Our initial search for precedents and analogues for ISIS includes 50 prior case of similarly [...] fanatical, purpose-driven groups, including the Bolsheviks of the Russian Revolution. Deciding which characteristic of ISIS we consider most salient - for example, its revolutionary politics or its religious millenarianism - helps us to narrow this list of the most instructive analogues.

\footnotetext{
${ }^{60}$ Applied History Project, URL: < http://www.belfercenter.org/project/applied-history-project > [consultato il 14 novembre 2017].

${ }^{61}$ ALLISON, Graham, FERGUSON, Niall, «Establish a White House Council of Historical Advisers Now», consultabile sul sito dell'AHP alla voce «Our Manifesto». Una versione condensata del testo è stata pubblicata sull'Atlantic con il titolo «Why the U.S. President Needs a Council of Historians», URL:

< https://www.theatlantic.com/magazine/archive/2016/09/dont-know-much-about-history/492746/ > [consultato 14 novembre 2017].

${ }^{62}$ Allison in particolare si è occupato di valutare le possibilità di un nuovo conflitto mondiale derivante da una degenerazione dei rapporti sino-americani cercando di valutare differenze e affinità tra lo scacchiere internazionale di oggi e quello del 1914. ALLISON, Graham, «Just How Likely Is Another World War?», in The Atlantic, 30 luglio 2014, URL: < https://www.theatlantic.com/international/archive/2014/07/just-howlikely-is-another-world-war/375320/ > [consultato il 14 novembre 2017].
} 
A systematic study of these other cases could help steer the president away from a potentially erroneous equation of ISIS with its most recent forerunner [al-Qaeda] ${ }^{63}$.

La scelta delle aree di ricerca, dunque, non è fine a se stessa ma si compie in relazione agli scenari internazionali e all'intenzione di rafforzare, integrandola con appropriate conoscenze storiche, la leadership americana nel mondo. Il potere esecutivo può inoltre trovare nella storia uno strumento di distensione nelle relazioni internazionali. Lo studio del passato, inteso come ricostruzione e comprensione della cultura e delle norme di una collettività nazionale, concorre a rafforzare «l'empatia tra culture» ${ }^{64}$ e può pertanto essere introdotto a pieno titolo in quell'insieme di strumenti e conoscenze che compongono il ricco apparato della diplomazia pubblica.

\section{Conclusioni}

Alla luce di quanto visto, è possibile affermare che il riavvicinamento proposto dalla public history e dalla applied history tra storia, opinione pubblica e istituzioni non è soltanto frutto di una «lotta corporativa per il potere, per quanto legittima e motivata» ${ }^{65}$, ma è soprattutto l'esito della riflessione che una parte della storiografia angloamericana ha condotto sullo statuto epistemologico della disciplina storica. I due indirizzi cercano di rispondere alla sfida che François Bédarida ha definito il moderno dilemma dello storico, ovvero «la scelta tra la torre d'avorio e la piazza del mercato»" ${ }^{66}$. Tra l'ipotesi di una storia accademica chiusa in se stessa, condannata all'irrilevanza, e una storia gettata nell'arena pubblica, public e applied history hanno provato a indicare una terza via, definendo una storiografia che sappia muoversi nel mondo e svolgere una funzione formativa senza perdere il proprio rigore e la propria identità scientifica.

Obiettività e autonomia rappresentano gli aspetti più controversi della public e dell'applied history. Le critiche principali derivano in parte da concreti problemi metodologici e in parte dalla difficoltà di definire l'ibridismo di due indirizzi che si collocano nel solco della storia tradizionale ma allo stesso tempo se ne allontanano, definendo nuovi criteri di ricerca e stabilendo rapporti con ambiti professionali eterogenei ${ }^{67}$. Il timore per la perdita di autonomia dello storico è avvertito specialmente per ciò che riguarda le relazioni tra storici e governi. Il rischio più

\footnotetext{
${ }^{63}$ ALLISON, Graham, FERGUSON, Niall, op. cit.

${ }^{64}$ Ibidem.

${ }^{65}$ STEVENS, Mary, «Public Policy and the Public Historian: The Changing Place of Historians in Public Life in France and the UK», in The Public Historian, 32, 3/2010, pp. 120-138, p. 124.

${ }^{66}$ BÉDARIDA, François, «The Modern Historian's Dilemma: Conflicting Pressures from Science and Society», in The Economic History Review, 40, 3/1987, pp. 335-348.

${ }^{67}$ STEARNS, Peter, «History and Policy Analysis: Toward Maturity», in The Public Historian, 4, 3/1982, pp. 429.
} 
evidente legato all'intersezione tra storia e politica è che potrebbe «inavvertitamente incoraggiare usi approssimativi o letture distorte del passato ${ }^{68}$, sebbene altri sottolineino una eventualità ancor peggiore ovvero che i governi, facendosi forti della collaborazione degli storici, ne approfittino per imporre una interpretazione ufficiale del passato, mettendo a repentaglio la libertà stessa del fare storia ${ }^{69}$. L'idea stessa che la storia debba essere impiegata per risolvere le contraddizioni del presente è stata negativamente giudicata tanto in Gran Bretagna che in America: Michael Oakeshott e Tony Judt - americano d'adozione - hanno criticato, in tempi diversi, la tendenza di certi storici a «sfruttare il passato per scopi attuali» e a «considerare la disciplina come un esercizio di polemica applicata ${ }^{70}$. Più di recente, sono stati espressi dei dubbi circa la possibilità di estrarre dallo studio del passato delle analogie o delle leggi oggettive per affiancare l'operato dei governi. In altre parole, la fattibilità stessa della applied history è stata messa in discussione ${ }^{71}$.

E tuttavia è proprio Judt che in un passo dedicato alla ricostruzione delle origini del pensiero neoliberista suggerisce implicitamente le potenzialità di un approccio storico alla politica. Il neoliberismo della scuola di Chicago, dimostra Judt, non è altro che il vecchio liberismo che un gruppo di intellettuali mitteleuropei portarono con sé nell'esilio americano:

We are the involuntary heirs to a debate with which most people are altogether unfamiliar [...] If we ask where the "Chicago boys" got their ideas, we shall find that the greatest influence was exercised by a handful of foreigners [...] Ludwig von Mises, Friedrich Hayek, Joseph Schumpeter, Karl Popper and Peter Drucker ${ }^{2}$.

Tutti e cinque svilupparono le proprie teorie circa i rapporti tra individuo, Stato e mercato alla luce della crisi dell'Austria liberale, apertasi con il fallimento dell'esperimento socialista di Vienna, aggravatasi con il fenomeno dell'austro-fascismo e conclusasi con l'Anschluss nel 1938.

Thus when we recapitulate conventional clichés about free markets and western liberties, we are in effect echoing - like light from a fading star - a debate inspired and conducted seventy years ago by men born for the most part in the late $19^{\text {th }}$ century [...] Most students in graduate business schools have never heard of some of these exotic foreign thinkers and are not

\footnotetext{
${ }^{68}$ STEVENS, Mary, op. cit., p. 131.

${ }^{69}$ BÉDARIDA, François, op. cit., p. 344. La possibilità che il potere politico fagociti la libertà d'espressione dello storico rappresenta un tema molto discusso dalla comunità storica francese ed è probabilmente la ragione principale dello scarso successo ottenuto dalla applied history in Francia. Si veda, ad esempio, l'attività del gruppo Liberté Pour l'Histoire, URL: < http://www.lph-asso.fr/ > [consultato il 4 aprile 2017]. ${ }^{70}$ JUDT, Tony, SNYDER, Timothy, Novecento, Roma-Bari, Laterza, 2012, p. 254.

${ }^{71}$ MORLEY, Neville, «Why Would Historians Make Bad Policy Advisers», in Aeon, 2 novembre 2016, URL:

< https://aeon.co/ideas/why-historians-would-make-bad-policy-advisers > [consultato il 15 novembre 2017].

${ }^{72}$ JUDT, Tony, Ill Fares the Land, New York, Penguin Press, 2010, pp. 97-98.
} 
encouraged to read them. And yet without an understanding of the Austrian origins of their (and our) way of thinking, it is as though we speak a language we do not fully comprehend ${ }^{73}$.

La applied history nelle sue formulazioni migliori sottolinea un principio a mio avviso implicito nel passo sopracitato, ovvero l'importanza di un pensiero pubblico e di un'azione politica storicizzati, consapevoli di svolgersi all'interno di una dimensione storica e in grado di utilizzare il contesto storico al fine di perfezionarsi. Tale è la forma di resistenza migliore che gli applied historian individuano contro gli usi impropri del passato.

\footnotetext{
${ }^{73}$ Ibidem, pp. 102-103.
} 


\section{L'AUTORE}

Benedetta GIULIANI ha conseguito nel 2016 la laurea magistrale in Scienze Storiche presso l'Università degli Studi di Roma La Sapienza. I suoi principali interessi di ricerca vertono sui rapporti tra Stati Uniti ed Europa e sulla storia dell'integrazione europea. Ha pubblicato articoli per le riviste «Eurostudium» e «Diacronie. Studi di Storia contemporanea». È attualmente iscritta al Master in International Public Affairs presso l'Università LUISS Guido Carli di Roma.

URL: < http://www.studistorici.com/progett/autori/\#Giuliani > 